\title{
Development and Clinical Validation of a Droplet Digital PCR Method for Detection of Acinetobacter baumannii and Klebsiella pneumonia in Patients with Suspected Bloodstream Infections
}

\author{
Yang Zheng ${ }^{1}$, Jun Jin ${ }^{1}$, Ziqiang Shao ${ }^{1}$, Jingquan Liu ${ }^{1}$, Run Zhang ${ }^{1}$, Renhua Sun ${ }^{1}$, and \\ Bangchuan $\mathrm{Hu}^{1}$ \\ 1Zhejiang Provincial People’s Hospital
}

June 11, 2021

\begin{abstract}
The relatively long turnaround time and low sensitivity of traditional blood culture may delay the effective antibiotic therapy in patients with bloodstream infection (BSI). To reduce the morbidity and mortality of BSI, a rapid and sensitive pathogen detection method is urgently required. Acinetobacter baumannii and Klebsiella pneumonia are two major microorganisms responsible for BSI. Here we reported a novel droplet digital PCR (ddPCR) method that can detect A. baumannii and K. pneumonia in whole blood samples within $4 \mathrm{~h}$, with a specificity of $100 \%$ for each strain and limit of detection at 0.93 copies/microliter for A. baumannii and 0.27 copies/microliter for K. pneumonia. Clinical validation in 170 patients with suspected BSIs showed that, compared with blood culture that reported $4(2.4 \%)$ A. baumannii cases and 7 (4.1\%) K. pneumonia cases, ddPCR detected $23(13.5 \%)$ A. baumannii cases, $26(15.3 \%) \mathrm{K}$. pneumonia cases, and $4(2.4 \%)$ dual infection cases, including the 11 positive patients reported by blood culture. In addition, the positive patients reported by ddPCR alone $(\mathrm{n}=$ 42) had significantly lower serum concentrations of procalcitonin and lactate, SOFA and APACHE II scores, and 28-day mortality than those reported by both blood culture and ddPCR $(n=11)$, suggesting that patients with less severe manifestations can potentially benefit from the guidance of ddPCR results. In conclusion, our study suggests that ddPCR represents a sensitive and rapid method to identify causal pathogens in blood samples and to guide the treatment decisions in the early stage of BSI.
\end{abstract}

Development and Clinical Validation of a Droplet Digital PCR Method for Detection of Acinetobacter baumannii andKlebsiella pneumonia in Patients with Suspected Bloodstream Infections

Yang Zheng ${ }^{1}$ \#, Jun Jin $^{1}$ \#, Ziqiang Shao ${ }^{1}$, Jingquan Liu $^{1}$, Run Zhang ${ }^{1}$,

Renhua $\mathrm{Sun}^{1}$, Bangchuan $\mathrm{Hu}^{1 *}$

${ }^{1}$ Intensive Care Unit, Zhejiang Provincial People's Hospital, People's Hospital of Hangzhou Medical College, 158 Shangtang Road, Hangzhou 310014, China.

\# These two authors contributed equally to this work.

Correspondence to:

Bangchuan Hu, PhD Zhejiang Provincial People's Hosital, Shangtang Road 158, Hangzhou 310014, China, Tel: +86-571-85893281, Fax:+86-571-85335800, E-mail:hubangchuanicu@163.com

\section{Abstract}

The relatively long turnaround time and low sensitivity of traditional blood culture may delay the effective antibiotic therapy in patients with bloodstream infection (BSI). To reduce the morbidity and mortality of BSI, a rapid and sensitive pathogen detection method is urgently required. Acinetobacter baumannii and 
Klebsiella pneumoniaare two major microorganisms responsible for BSI. Here we reported a novel droplet digital PCR (ddPCR) method that can detect A. baumannii and $K$. pneumonia in whole blood samples within $4 \mathrm{~h}$, with a specificity of $100 \%$ for each strain and limit of detection at 0.93 copies/microliter for $A$. baumannii and 0.27 copies/microliter for K. pneumonia. Clinical validation in 170 patients with suspected BSIs showed that, compared with blood culture that reported 4 (2.4\%) A. baumannii cases and $7(4.1 \%)$ K. pneumonia cases, ddPCR detected 23 (13.5\%) A. baumannii cases, $26(15.3 \%)$ K. pneumonia cases, and $4(2.4 \%)$ dual infection cases, including the 11 positive patients reported by blood culture. In addition, the positive patients reported by ddPCR alone $(\mathrm{n}=42)$ had significantly lower serum concentrations of procalcitonin and lactate, SOFA and APACHE II scores, and 28-day mortality than those reported by both blood culture and ddPCR $(\mathrm{n}=11)$, suggesting that patients with less severe manifestations can potentially benefit from the guidance of ddPCR results. In conclusion, our study suggests that ddPCR represents a sensitive and rapid method to identify causal pathogens in blood samples and to guide the treatment decisions in the early stage of BSI.

Keywords: Acinetobacter baumannii, Klebsiella pneumonia,droplet digital PCR, Bloodstream infection, pathogen

\section{Introduction}

Bloodstream infection (BSI) represents a major cause of death worldwide, contributing to increased healthcare costs, length of hospital stay, and in-hospital morbidity (McNamara, et al., 2018). Timely and accurate pathogen identification is critical to guide antimicrobial treatment for patients in the early stage of BSI. Blood culture remains the gold standard for identifying the pathogens in BSI (Blevins and Bronze, 2010). However, it is limited by the low sensitivity and the long turnaround time (Riedel and Carroll, 2016, Tabak, et al., 2018). In septic patients within the first $6 \mathrm{~h}$ of documented hypotension, every 1-h delay in appropriate antibiotic therapy leads to an average increase of mortality rate by $7.6 \%$ (Kumar, et al., 2006). For hospitalized patients with bacterial infections, inappropriate initial antimicrobial treatment almost doubles the risk of 30-day mortality (Fraser, et al., 2006). Thus, it is necessary to develop a rapid and accurate method to identify the causal pathogens in BSI.

Recently, the culture-independent, real-time PCR-based or microarray-based methods, such as SeptiFast (Roche, Switzerland), Magicplex (Seegene, Korea), and TaqMan array card assay (Academy of Military Medical Science, China), have shown promising performance in rapidly identifying the pathogens and initiating early targeted antibiotic therapy in BSI. However, the low sensitivities ranging from $29 \%$ to $79.4 \%$ may limit the clinical application of these methods (Warhurst, et al., 2015, Buehler, et al., 2016, Riedel and Carroll, 2016, Zhang, et al., 2018, Zboromyrska, et al., 2019). Droplet digital polymerase chain reaction (ddPCR) is a novel molecular method to detect and quantify nucleic acids. In ddPCR, the template is partitioned into thousands of nanoliter-sized droplets and amplified. After amplification, the numbers of positive and negative reactions are counted, and the copy number of the template is calculated using Poisson statistics (Huggett, et al., 2015, Kuypers and Jerome, 2017). As an emerging versatile tool with high sensitivity, accuracy, and precision, ddPCR has been increasingly applied in multiple clinical scenarios, including oncology (Gevensleben, et al., 2013, Taly, et al., 2013, Jennings, et al., 2014, Postel, et al., 2018, Galimberti, et al., 2019), non-invasive prenatal testing (Barrett, et al., 2012, Tan, et al., 2019), and infectious diseases (Kelley, et al., 2013, Pholwat, et al., 2013, Sedlak, et al., 2014, Sedlak, et al., 2014, Whale, et al., 2016, Wouters, et al., 2019).

Acinetobacter baumannii and Klebsiella pneumoniae are two major Gram-negative bacteria involved in BSI, with high capabilities to develop antibiotic resistance. BSIs due to multidrug-resistant A. baumannii and K. pneumoniae significantly contribute to the mortality in the intensive care unit (ICU), with a mortality rate over 50\% (Balkhair, et al., 2019, Brink, 2019). In this study, we developed and validated a ddPCRbased method to detect A.baumannii and K. pneumonia in blood samples of patients with suspected BSI. Our results provide ddPCR as a promising method to accurately and rapidly diagnose BSIs caused by $A$. baumannii and K. pneumoniae. 


\section{Materials and methods}

\section{Blood culture and control bacterial strains}

Upon clinical suspicion of BSI, whole blood samples were simultaneously obtained for blood culture and molecular diagnosis. Two sets of blood cultures were collected for each patient according to routine clinical practice, and each set consisted of an aerobic bottle and an anaerobic bottle. The blood cultures were incubated at 37 in a BacT/ALERT@ 3D System (BioMérieux, France). When the system showed a positive signal, Gram staining was performed, followed by subculture on a Columbia blood agar plate at 37 with $5 \% \mathrm{CO}_{2}$. Following overnight incubation, the pathogens were further identified by matrix-assisted laser desorption-ionization time-of-flight mass spectrometry (MALDI-TOF MS) (VITEK(r) MS system, BioMerieux, France). The positive control bacteria were A. baumannii ATCC 19606 with 5 clinical isolates and K. pneumonia CMCC 46117 with 33 clinical isolates. A total of 131 other clinical isolates that are commonly found in BSI were used as negative controls (Table 1 ).

\section{DNA extraction}

Total DNA was isolated from $1 \mu \mathrm{L}$ of purified control bacterial strains using a TIANamp bacteria DNA kit (TIANGEN Biotech, Beijing, China) following the manufacturer's instructions. Plasma was obtained by centrifuging the blood sample at 1,600 $\times \mathrm{g}$ for $20 \mathrm{~min}$. Plasma DNA extraction was isolated from $2 \mathrm{~mL}$ plasma using a magnetic serum/plasma DNA kit (TIANGEN Biotech, Beijing, China) and the Auto-Pure20B nucleic acid purification system (Hangzhou Allsheng Instruments, Zhejiang, China). DNA was eluted in 50 $\mu \mathrm{L}$ of elution buffer, and stored at $-80^{\circ} \mathrm{C}$ until use.

\section{Primers and probes}

Primers and TaqMan MGB probes (Table 2 ) were designed using Primer Express (Thermo Fisher Scientific, Waltham, MA, USA) and synthesized by General Biosystems (Chuzhou, China). A ROX or Cy5 reporter was incorporated at the 5' end of the probes, and a nonfluorescent quencher at the 3' end. The sensitivity and specificity were evaluated using the sequence alignments in GenBank and the Basic Local Alignment Search Tool on NCBI, respectively.

\section{Droplet digital PCR}

ddPCR was carried out using a ddPCR platform (Pilot Gene Technology, Hangzhou, China). A duplex ddPCR assay was performed to detect $A$. baumannii and $K$. pneumonia simultaneously in one chip. ddPCR analysis was performed using a Pilot Gene Droplet Digital PCR System following the manufacturer's protocol. Briefly, the ddPCR master mix for each testing panel had a final volume of $15 \mu \mathrm{L}$ and contained $1 \times$ ddPCR premix, $1 \mu \mathrm{M}$ forward and reverse primers, $300 \mathrm{nM}$ each probe, $5 \mu \mathrm{L}$ of isolated plasma DNA, and DNasefree water. The mixture was loaded on a ready-to-use disposable plastic chip. About 20,000 droplets were generated using a droplet generator (DG32; Pilot Gene Technology), followed by amplification on a TC1 thermal cycler (Pilot Gene Technology). The thermal cycling parameters were $95{ }^{\circ} \mathrm{C}$ for 5 min followed by 40 cycles of $95{ }^{\circ} \mathrm{C}$ for $15 \mathrm{~s}$ and $60{ }^{\circ} \mathrm{C}$ for $60 \mathrm{~s}$. After PCR amplification, droplets were analysed using an iScanner 5 chip scanner. Data analysis for droplet counts and amplitudes was performed with 30 min of hands-on time using GenePMS software version v2.0.01.20011.

\section{Evaluation of specificity, sensitivity, linearity, and precision}

Analytical specificity was evaluated by testing genomic DNA isolated from the positive and negative control bacterial strains. Analytical sensitivity was determined using LoB and LoD assays with samples in 22 replicates. Probit analysis was performed to measure $\mathrm{LoD}$ for each bacterium. The mean value of the copy number and the standard deviation (SD) were calculated. $\mathrm{LoB}$ was calculated as mean blank $+1.645\left(\mathrm{SD}_{\text {blank }}\right)$. $\mathrm{LoD}$ was calculated as mean blank $+3\left(\mathrm{SD}_{\text {blank }}\right)$ (Armbruster and Pry, 2008). The linearity was determined by a 2 -fold serial dilution of the DNA template, and each dilution was measured in 8 replicates. Precision was evaluated by testing five different concentrations of genomic DNA in 8 replicates.

\section{Patients}


A total of 170 patients were recruited from Zhejiang Provincial People's Hospital from March 2019 to October 2020. The inclusion criteria were age $>18$ years and suspicion of BSI. Suspected BSI was defined if the patient had a sudden high fever $\left(\mathrm{T}[?] 38.5^{\circ} \mathrm{C}\right)$ accompanied by hemodynamic instability that could not be explained by a site-specific infection at another body site. In addition, the recruited patient also presented life-threatening organ dysfunction with an increase of 2 points or more in the sepsis-related organ failure assessment score (Warhurst, et al., 2015, Timsit, et al., 2020). The demographic and clinical characteristics of each patient were collected within the first $24 \mathrm{~h}$ of suspicion of BSI and summarized inTable 3 . Telephone follow-up interviews were conducted with surviving patients. An unfavorable outcome was defined as 28-day all-cause mortality after ICU admission. This study was approved by the Institutional Review Board and the Ethics Committee of Zhejiang Provincial People's Hospital (No. 2019KY002). All patients provided informed written consent.

\section{Statistical analysis}

SAS 9.13 (SAS Institute, NC, USA) was used for database management and statistical analyses. Continuous variables were expressed as the mean and standard deviation (SD) or median and interquartile range (IQR) where appropriate. The t test was used to analyze normally distributed continuous variables, whereas the Mann-Whitney U test was used to analyze nonnormally distributed continuous variables. Categorical variables were reported as frequencies and percentages, and analyzed using the chi-square test. $P$ values less than 0.05 were considered statistically significant.

\section{Results}

\section{Analytical specificity}

To detect A. baumannii and $K$. pneumonia in blood samples, we designed a specific primer-probe set for each strain. The specificity test showed that all $5 \mathrm{~A}$. baumannii isolates and $33 \mathrm{~K}$. pneumonia isolates from ATCC were detected by corresponding primer-probe set, whereas the 131 negative control isolates showed no response to the primer-probe sets (Table 4 ). These results suggest that the primer-probe sets are specific to A. baumanniiand K. pneumonia, respectively.

\section{Analytical sensitivity}

Analytical sensitivity was measured using the limit of blank (LoB) and limit of detection (LoD) assays. As shown in Table $4, \mathrm{LoB}$ of $A$. baumannii and K. pneumonia were 0.55 copies/ $\mu \mathrm{L}$ and 0.15 copies $/ \mu \mathrm{L}$, respectively; LoD of $A$. baumannii and K. pneumonia were 0.93 copies/ $\mu \mathrm{L}$ and 0.27 copies/ $\mu \mathrm{L}$, respectively. LoD served as the lowest concentration that gave positive results in the ddPCR assay.

\section{Analytical repeatability, reproducibility, and linearity}

Within-run precision (repeatability) and within-laboratory precision (reproducibility) were measured using different concentrations of sheared A. baumannii and $K$. pneumonia DNA samples. The within-run coefficients of variations (CVs) of $A$. baumannii and $K$. pneumonia were $2.7 \%-5.3 \%$ and $2.3 \%-9.5 \%$, respectively. The within-laboratory CVs of $A$. baumannii and K. pneumoniawere $5.3 \%-7.5 \%$ and $7.4 \%-9.7 \%$, respectively (Tables 5 and 6).

Linearity was determined by a 2 -fold serial dilution of the DNA template. Figure 1 shows the regression lines that represent the linear relationships between DNA copy numbers and concentrations of $A$. baumannii $\left(R^{2}=0.9925\right)$ and $K$. pneumonia $\left(R^{2}=0.9915\right)$, respectively. Taken together, these results indicate that ddPCR has excellent repeatability, reproducibility, and linearity in detecting A. baumannii and K. pneumonia in blood samples.

\section{Turnaround time of diagnosis}

The blood samples were obtained and transferred to ddPCR laboratory in about $10 \mathrm{~min}$. Plasma was immediately isolated after centrifugation for $20 \mathrm{~min}$. DNA extraction and PCR amplification were completed within $3 \mathrm{~h}$. Data analysis was done within $30 \mathrm{~min}$ using GenePMS software. The average turnaround time 
of ddPCR was $4.2 \pm 0.51 \mathrm{~h}$, which was remarkably shorter than that of the blood culture test $(90.6 \pm 10.9$ h, $P<0.01)$.

\section{Clinical performance}

Of the 170 patients with suspected BSIs tested by ddPCR, 53 showed positive results, including 23 (13.5\%, $23 / 170)$ A. baumanniicases, $26(15.3 \%, 26 / 170) K$. pneumonia cases, and $4(2.4 \%, 4 / 170)$ dual infection cases. On the other hand, blood culture detected $4(2.4 \%)$ A. baumannii cases, 7 (4.1\%) K. pneumoniacases, and $22(12.9 \%)$ cases with other bacterial infections, with an overall positivity rate of $19.4 \%(33 / 170)$. Of note, all the 11 cases $(100 \%, 11 / 11)$ with A. baumannii or K. pneumoniainfections reported by blood culture showed positive results in ddPCR assay. The positivity rate of A. baumannii and K. pneumonia in the ddPCR assay was much higher than that in the blood culture $(31.2 \%, 53 / 170$ vs. $6.5 \%, 11 / 170)$ (Table 7 ).

The clinical characteristics of the 53 ddPCR-positive patients were summarized in Table 3 . No significant differences were observed in age, systolic and diastolic blood pressure, the plasma level of CRP, white blood cell counts, serum creatinine, and use of vasoactive drugs (all $P$ [?] 0.05) between the patients reported by ddPCR alone $(\mathrm{n}=42)$ and the patients reported by both ddPCR and blood culture $(\mathrm{n}=11)$. Importantly, compared with the 11 positive patients reported by both ddPCR and blood culture, the 42 positive patients reported by ddPCR alone had significantly decreased serum concentrations of procalcitonin (3.70 vs. 12.9 $\mathrm{pg} / \mathrm{L}, P=0.03)$ and lactate $(2.76$ vs. $4.26 \mathrm{mmol} / \mathrm{L}, P=0.04)$, APACHE II scores $(21.9$ vs. $27.9, P=0.02)$, SOFA scores $(10.5$ vs. $13.8, P=0.03)$, and 28 -day mortality rates $(70.6 \%$ vs. $90.1 \%, P=0.04)$ (Table 3 ). These data suggest that the less severe BSI patients who have been missed by blood culture may benefit from ddPCR test and have better clinical outcomes.

\section{Discussion}

By studying 165,593 blood specimens from 13 USA hospitals, Tabak et al. have shown that the median time to identify BSI pathogens using traditional blood culture was $44.0 \mathrm{~h}$, with a sensitivity of approximately $70 \%$ in critically ill patients and even lower for fastidious microorganisms (Tabak, et al., 2018). In this study, to overcome the shortcomings of the blood culture in BSI diagnosis, we developed a culture-independent ddPCR method to rapidly and accurately identify A. baumannii and K. pneumonia in blood samples of patients with suspected BSI. Our results showed that ddPCR could identify A. baumannii and K. pneumonia in whole blood samples within $4 \mathrm{~h}$, with a specificity of $100 \%$ for each strain and limit of detection at 0.93 copies $/ \mu \mathrm{L}$ for A. baumannii and 0.27 copies/ $\mu \mathrm{L}$ for K. pneumonia. Clinical validation in 170 patients with suspected BSIs showed that ddPCR not only detected the positive patients who were identified by blood culture but also detected the patients who were missed by blood culture. Of note, compared with the patients reported by both blood culture and ddPCR assay, the patients reported by ddPCR alone had less severe clinical manifestations and better clinical outcomes, suggesting that these patients benefit from the guidance of ddPCR results in the early stage of BSI. Thus, ddPCR may serve as a rapid and reliable method to identify causal pathogens in BSI and to guide the treatment decisions in the early stage of BSI.

In BSI, the human immune system and antibiotic treatment kill invading pathogens, leading to the release of the nucleic acids from the pathogens into the blood, which become a part of circulating cell-free DNA (cfDNA) (Lo, et al., 1999, Diehl, et al., 2008, Lu, et al., 2018, Papadopoulos, 2020). Thus, the presence of specific pathogenic DNA in cfDNA can reflect the presence of pathogens in the bloodstream. Accumulating evidence have demonstrated the feasibility of next-generation sequencing (NGS) of plasma cfDNA to identify the pathogens in BSI (Blauwkamp, et al., 2019, Farnaes, et al., 2019, Grumaz, et al., 2019, Rossoff, et al., 2019). However, the typical turnaround time of 2 days and the high cost of NGS remain barriers for cfDNA NGS in clinical practice (Long, et al., 2016, Simner, et al., 2018, Chiu and Miller, 2019, Crawford, et al., 2019). In this study, we took advantage of the ultra-high sensitivity of ddPCR and the feasibility of cfDNA in pathogen identification to develop a ddPCR-based method using cfDNA as the template. The turnaround time of ddPCR to diagnosis was $4 \mathrm{~h}$, which was significantly shorter than that of NGS $(2-3 \mathrm{~d})$ or blood culture (90.6 $\pm 12.9 \mathrm{~h}$ in this study). 
Wouters et al. have developed a ddPCR method to detect bacteria or fungi using metagenomic DNA as the template and broad-range primer-probe sets; however, the overall specificity in clinical validation was only $80 \%$ (Wouters, et al., 2019). In this study, we used cfDNA as the template and designed specific primer-probe sets for A. baumannii and K. pneumonia, respectively. We achieved $100 \%$ specificity in well-characterized ATCC isolates of each strain, which was also higher than other reported PCR-based methods, such as SeptiFast (50\%, (Warhurst, et al., 2015); 85.5\%, (Korber, et al., 2017)), Magicplex (29\%) (Zboromyrska, et al., 2019), TAC assay (79.4\%) (Zhang, et al., 2018), T2Bacteria (90\%) (Maki, 2019, Nguyen, et al., 2019), and cfDNA NGS $(93.7 \%)$ (Blauwkamp, et al., 2019).

The sensitivity of blood culture is typically lower than expected. The blood culture positivity rate of septic patients was $51 \%$ over 22 years in the United States (Martin, et al., 2003). Cheng et al. have reported a 71.7\% blood culture positivity rate in severe sepsis patients from ten university hospitals in China (Cheng, et al., 2007). Similarly, $70 \%$ of infected patients in a one-day international investigation in ICU have been reported positive by blood culture test (Vincent, et al., 2009). The low sensitivity of blood culture may attribute to the low bacteria abundance in the blood, the antibiotic treatment before sampling, and the culture techniques. Molecular detection methods are less affected by these factors, thus usually have higher positivity rates than blood culture. The positivity rates of different molecular methods are 1.56 - 6.45 -fold higher than that of blood culture (Long, et al., 2016, Korber, et al., 2017, Farnaes, et al., 2019, Grumaz, et al., 2019, Nguyen, et al., 2019). In the present study, the positivity rates of A. baumannii and K. pneumonia were 6.8 -fold $((23+4) / 4)$ and 4.3 -fold $((26+4) / 7)$ higher than those of blood culture, respectively. Thus, molecular detection methods may disclose the missed diagnosis in blood culture, allowing timely diagnosis and appropriate antibiotic treatment for the patients with BSI.

In this study, the 53 positive patients detected by ddPCR had typical manifestations resulting from BSI, including body temperature greater than 38.5, abnormally elevated serum levels of C-reactive protein and procalcitonin, hemodynamic instability, and severe organ dysfunction. Of note, the positive patients reported by ddPCR alone exhibited less severe manifestations than those reported by both ddPCR and blood culture, suggesting that ddPCR is more sensitive than blood culture for early diagnosis of BSI.

In conclusion, we developed a novel ddPCR method to detect two major pathogens in patients with suspected BSI. Clinical validation revealed that our method outperformed the blood culture in specificity, sensitivity, and turnaround time, serving as a promising method for early and accurate diagnosis of BSI. However, in this pilot study, we only included two major Gram-negative bacteria responsible for BSI. More clinically important pathogens will be further investigated in future studies.

\section{Acknowledgment}

We gratefully acknowledge Dr. Seng Ye and Dr. Renyang Liu for their assistance with the project. We also thank Dr. Xianghong Yang for the useful discussion.

\section{Funding}

The study was financially supported by grants from Key Research and Development Project of the Science Technology Department of Zhejiang Province (2020C03031), National Natural Science Foundation of China (81971857), and the Natural Science Foundation of Zhejiang Province (LY17H15000).

\section{Competing interests}

All authors declare that they have no competing interests.

\section{Author contributions}

Yang Zheng Jun Jing and Bangchuan Hu conceived the study design, analyzed and interpreted the data, and drafted the manuscript. Ziqiang Shao and Renhua Sun performed research and contributed analytic tools. Jingquan Liu and Run Zhang participated in study design, acquired the data, and helped revise the manuscript. All authors read and approved the final manuscript. 


\section{Ethics statement}

The study protocol was approved by the Institutional Review Board and Ethics Committee (No. 2019KY002) of Zhejiang Provincial People's Hospital, and adhered to the guidelines of Institutional Review Board and Ethics Committee.

\section{References}

Armbruster, D.A., and Pry, T. (2008) Limit of blank, limit of detection and limit of quantitation. Clin Biochem Rev, 29 (Suppl 1), S49-S52.

Balkhair, A., Al-Muharrmi, Z., Al'Adawi, B., Al Busaidi, I., Taher, H.B., Al-Siyabi, T., et al. (2019) Prevalence and 30-day all-cause mortality of carbapenem-and colistin-resistant bacteraemia caused by Acinetobacter baumannii, Pseudomonas aeruginosa, and Klebsiella pneumoniae: Description of a decade-long trend, Int J Infect Dis,85 , 10-15. https://doi: 10.1016/j.ijid.2019.05.004.

Barrett, A.N., McDonnell, T.C.R., Chan, K.C.A., and Chitty, L.S. (2012) Digital PCR Analysis of Maternal Plasma for Noninvasive Detection of Sickle Cell Anemia, Clinical Chemistry, 58 (6), 1026-1032. https://doi: 10.1373/clinchem.2011.178939.

Blauwkamp, T.A., Thair, S., Rosen, M.J., Blair, L., Lindner, M.S., Vilfan, I.D., et al. (2019) Analytical and clinical validation of a microbial cell-free DNA sequencing test for infectious disease. Nat Microbiol, 4(4), 663-674. https://doi: 10.1038/s41564-018-0349-6.

Blevins, S.M., and Bronze, M.S. (2010) Robert Koch and the 'golden age' of bacteriology. International Journal of Infectious Diseases, 14(9), e744-e751. https://doi: 10.1016/j.ijid.2009.12.003.

Brink, A.J. (2019) Epidemiology of carbapenem-resistant Gram-negative infections globally. Curr Opin Infect Dis, 32(6), 609-616. https://doi: 10.1097/QCO.0000000000000608.

Buehler, S.S., Madison, B., Snyder, S.R., Derzon, J.H., Cornish, N.E., Saubolle, M.A., et al. (2016) Effectiveness of Practices To Increase Timeliness of Providing Targeted Therapy for Inpatients with Bloodstream Infections: a Laboratory Medicine Best Practices Systematic Review and Meta-analysis. Clinical Microbiology Reviews, 29(1), 59-103. https://doi: 10.1128/CMR.00053-14.

Cheng, B., Xie, G., Yao, S., Wu, X., Guo, Q., Gu, M., et al. (2007) Epidemiology of severe sepsis in critically ill surgical patients in ten university hospitals in China. Crit Care Med, 35(11), 2538-2546. https://doi:10.1097/01.CCM.0000284492.30800.00.

Chiu, C.Y., and Miller, S.A. (2019) Clinical metagenomics. Nat Rev Genet, 20(6), 341-355. https://doi: 10.1038/s41576-019-0113-7.

Crawford, E., Kamm, J., Miller, S., Li, L.M., Caldera, S., Lyden, A., et al. (2019) Investigating TransfusionRelated Sepsis using Culture-Independent Metagenomic Sequencing. Clin Infect Dis, 71(5),1179-1185. https://doi: 10.1093/cid/ciz960.

Diehl, F., Schmidt, K., Choti, M.A., Romans, K., Goodman, S., Li, M., et al. (2008) Circulating mutant DNA to assess tumor dynamics. Nat Med, 14(9), 985-990. https://doi: 10.1038/nm.1789.

Farnaes, L., Wilke, J., Ryan Loker, K., Bradley, J.S., Cannavino, C.R., Hong, D.K., et al. (2019) Communityacquired pneumonia in children: cell-free plasma sequencing for diagnosis and management. Diagn Microbiol Infect Dis, 94(2), 188-191. https://doi: 10.1016/j.diagmicrobio.2018.12.016.

Fraser, A., Paul, M., Almanasreh, N., Tacconelli, E., Frank, U., Cauda, R., et al. (2006) Benefit of Appropriate Empirical Antibiotic Treatment: Thirty-day Mortality and Duration of Hospital Stay. The American Journal of Medicine, 119(11), 970-976. https://doi: 10.1016/j.amjmed.2006.03.034.

Galimberti, S., Genuardi, E., Mazziotta, F., Iovino, L., Morabito, F., Grassi, S., et al. (2019) The Minimal Residual Disease in Non-Hodgkin's Lymphomas: From the Laboratory to the Clinical Practice. Frontiers in 
Oncology, 9, 528. https://doi: 10.3389/fonc.2019.00528.

Gevensleben, H., Garcia-Murillas, I., Graeser, M.K., Schiavon, G., Osin, P., Parton, M., et al. (2013) Noninvasive Detection of HER2 Amplification with Plasma DNA Digital PCR. Clinical Cancer Research, 19(12), 3276-3284. https://doi: 10.1158/1078-0432.CCR-12-3768.

Grumaz, S., Grumaz, C., Vainshtein, Y., Stevens, P., Glanz, K., Decker, S.O., et al. (2019) Enhanced Performance of Next-Generation Sequencing Diagnostics Compared With Standard of Care Microbiological Diagnostics in Patients Suffering From Septic Shock. Crit Care Med, 47(5), e394-e402. https://doi: 10.1097/CCM.0000000000003658.

Huggett, J.F., Cowen, S., and Foy, C.A. (2015) Considerations for Digital PCR as an Accurate Molecular Diagnostic Tool. Clinical Chemistry, 61(1), 79-88. https://doi: 10.1373/clinchem.2014.221366.

Jennings, L.J., George, D., Czech, J., Yu, M., and Joseph, L. (2014) Detection and Quantification of BCRABL1 Fusion Transcripts by Droplet Digital PCR. The Journal of Molecular Diagnostics, 16(2), 174-179. https://doi: 10.1016/j.jmoldx.2013.10.007.

Kelley, K., Cosman, A., Belgrader, P., Chapman, B., and Sullivan, D.C. (2013) Detection of MethicillinResistant Staphylococcus aureus by a Duplex Droplet Digital PCR Assay. Journal of Clinical Microbiology, 51(7), 2033-2039. https://doi: 10.1128/JCM.00196-13.

Korber, F., Zeller, I., Grunstaudl, M., Willinger, B., Apfalter, P., Hirschl, A.M., and Makristathis, A. (2017) SeptiFast versus blood culture in clinical routine - A report on 3 years experience. Wien Klin Wochenschr, 129(11-12), 427-434. https://doi: 10.1007/s00508-017-1181-3.

Kumar, A., Roberts, D., Wood, K.E., Light, B., Parrillo, J.E., Sharma, S., et al. (2006) Duration of hypotension before initiation of effective antimicrobial therapy is the critical determinant of survival in human septic shock. Critical Care Medicine, 34(6), 1589-1596. https://doi: 10.1097/01.CCM.0000217961.75225.E9.

Kuypers, J., and Jerome, K.R. (2017) Applications of Digital PCR for Clinical Microbiology. Journal of Clinical Microbiology, 55(6), 1621-1628. https://doi: 10.1128/JCM.00211-17.

Lo, Y.M., Zhang, J., Leung, T.N., Lau, T.K., Chang, A.M., and Hjelm, N.M. (1999) Rapid clearance of fetal DNA from maternal plasma. Am J Hum Genet, 64(1), 218-224. https://doi: 10.1086/302205.

Long, Y., Zhang, Y., Gong, Y., Sun, R., Su, L., Lin, X., et al. (2016) Diagnosis of Sepsis with Cell-free DNA by Next-Generation Sequencing Technology in ICU Patients. Arch Med Res, 47(5), 365-371. https://doi: 10.1016/j.arcmed.2016.08.004.

Lu, L., Bi, J., and Bao, L. (2018) Genetic profiling of cancer with circulating tumor DNA analysis. J Genet Genomics, 45(2), 79-85. https://doi: 10.1016/j.jgg.2017.11.006.

Maki, D.G. (2019) The T2Bacteria Panel had $90 \%$ sensitivity for detecting targeted organisms, $43 \%$ for any bloodstream infection organism. Ann Intern Med, 171(6), JC34. https://doi: 10.7326/ACPJ201909170-034.

Martin, G.S., Mannino, D.M., Eaton, S., and Moss, M. (2003) The epidemiology of sepsis in the United States from 1979 through 2000. N Engl J Med, 348(16), 1546-1554. https://doi: 10.1056/NEJMoa022139.

McNamara, J.F., Righi, E., Wright, H., Hartel, G.F., Harris, P.N.A., and Paterson, D.L. (2018) Long-term morbidity and mortality following bloodstream infection: A systematic literature review. J Infect, 77(1), 1-8. https://doi: 10.1016/j.jinf.2018.03.005.

Nguyen, M.H., Clancy, C.J., Pasculle, A.W., Pappas, P.G., Alangaden, G., Pankey, G.A., et al. (2019) Performance of the T2Bacteria Panel for Diagnosing Bloodstream Infections: A Diagnostic Accuracy Study. Ann Intern Med, 170(12), 845-852. https://doi: 10.7326/M18-2772.

Papadopoulos, N. (2020) Pathophysiology of ctDNA Release into the Circulation and Its Characteristics: What Is Important for Clinical Applications. Recent Results Cancer Res, 215, 163-180. https://doi: 


\subsection{7/978-3-030-26439-0_9.}

Pholwat, S., Stroup, S., Foongladda, S., and Houpt, E. (2013) Digital PCR to Detect and Quantify Heteroresistance in Drug Resistant Mycobacterium tuberculosis. PLOS ONE, 8(2), e57238. https://doi: 10.1371/journal.pone.0057238.

Postel, M., Roosen, A., Laurent-Puig, P., Taly, V., and Wang-Renault, S.-F. (2018) Droplet-based digital PCR and next generation sequencing for monitoring circulating tumor DNA: a cancer diagnostic perspective. Expert Review of Molecular Diagnostics, 18(1), 7-17. https://doi: 10.1080/14737159.2018.1400384.

Riedel, S., and Carroll, K.C. (2016) Early Identification and Treatment of Pathogens in Sepsis: Molecular Diagnostics and Antibiotic Choice. Clinics in Chest Medicine, 37(2), 191-207. https://doi: 10.1016/j.ccm.2016.01.018.

Rossoff, J., Chaudhury, S., Soneji, M., Patel, S.J., Kwon, S., Armstrong, A., and Muller, W.J. (2019) Noninvasive Diagnosis of Infection Using Plasma Next-Generation Sequencing: A Single-Center Experience. Open Forum Infect Dis, 6(8), ofz327. https://doi: 10.1093/ofid/ofz327.

Sedlak, R.H., Cook, L., Cheng, A., Magaret, A., and Jerome, K.R. (2014) Clinical Utility of Droplet Digital PCR for Human Cytomegalovirus. Journal of Clinical Microbiology, 52(8), 2844-2848. https://doi: 10.1128/JCM.00803-14.

Sedlak, R.H., Cook, L., Huang, M.-L., Magaret, A., Zerr, D.M., Boeckh, M., and Jerome, K.R. (2014) Identification of Chromosomally Integrated Human Herpesvirus 6 by Droplet Digital PCR. Clinical Chemistry, 60(5), 765-772. https://doi: 10.1373/clinchem.2013.217240.

Simner, P.J., Miller, S., and Carroll, K.C. (2018) Understanding the Promises and Hurdles of Metagenomic Next-Generation Sequencing as a Diagnostic Tool for Infectious Diseases. Clin Infect Dis, 66(5), 778-788. https://doi: 10.1093/cid/cix881.

Tabak, Y.P., Vankeepuram, L., Ye, G., Jeffers, K., Gupta, V., and Murray, P.R. (2018) Blood Culture Turnaround Time in U.S. Acute Care Hospitals and Implications for Laboratory Process Optimization. Journal of Clinical Microbiology, 56(12), e00500-00518. https://doi: 10.1128/JCM.00500-18.

Taly, V., Pekin, D., Benhaim, L., Kotsopoulos, S.K., Le Corre, D., Li, X., et al. (2013) Multiplex Picodroplet Digital PCR to Detect KRAS Mutations in Circulating DNA from the Plasma of Colorectal Cancer Patients. Clinical Chemistry, 59(12), 1722-1731. https://doi: 10.1373/clinchem.2013.206359.

Tan, C., Chen, X., Wang, F., Wang, D., Cao, Z., Zhu, X., et al. (2019) A multiplex droplet digital PCR assay for non-invasive prenatal testing of fetal aneuploidies. Analyst, 144(7), 2239-2247. https://doi: $10.1039 / \mathrm{c} 8 \mathrm{an} 02018 \mathrm{c}$.

Timsit, J.F., Ruppe, E., Barbier, F., Tabah, A., and Bassetti, M. (2020) Bloodstream infections in critically ill patients: an expert statement. Intensive Care Med, 46(2), 266-284. https://doi: 10.1007/s00134-02005950-6.

Vincent, J.L., Rello, J., Marshall, J., Silva, E., Anzueto, A., Martin, C.D., et al. (2009) International study of the prevalence and outcomes of infection in intensive care units. JAMA, 302(21), 2323-2329. https://doi: 10.1001/jama.2009.1754.

Warhurst, G., Dunn, G., Chadwick, P., Blackwood, B., McAuley, D., Perkins, G.D., et al. (2015) Rapid detection of health-care-associated bloodstream infection in critical care using multipathogen real-time polymerase chain reaction technology: a diagnostic accuracy study and systematic review. Health Technol Assess, 19(35),1-142. https:// doi: 10.3310/hta19350.

Warhurst, G., Maddi, S., Dunn, G., Ghrew, M., Chadwick, P., Alexander, P., et al. (2015) Diagnostic accuracy of SeptiFast multi-pathogen real-time PCR in the setting of suspected healthcare-associated bloodstream infection. Intensive Care Med, 41(1), 86-93. https://doi: 10.1007/s00134-014-3551-x. 
Whale, A.S., Bushell, C.A., Grant, P.R., Cowen, S., Gutierrez-Aguirre, I., O'Sullivan, D.M., et al. (2016) Detection of Rare Drug Resistance Mutations by Digital PCR in a Human Influenza A Virus Model System and Clinical Samples. Journal of Clinical Microbiology, 54(2), 392-400. https://doi: 10.1128/JCM.02611-15.

Wouters, Y., Dalloyaux, D., Christenhusz, A., Roelofs, H.M.J., Wertheim, H.F., Bleeker-Rovers, C.P., et al. (2020) Droplet digital polymerase chain reaction for rapid broad-spectrum detection of bloodstream infections. Microbial Biotechnology, 13(3), 657-668. https://doi: 10.1111/1751-7915.13491.

Zboromyrska, Y., Cilloniz, C., Cobos-Trigueros, N., Almela, M., Hurtado, J.C., Vergara, A., et al. (2019) Evaluation of the Magicplex Sepsis Real-Time Test for the Rapid Diagnosis of Bloodstream Infections in Adults. Front Cell Infect Microbiol, 9, 56. https://doi: 10.3389/fcimb.2019.00056.

Zhang, C., Zheng, X., Zhao, C., Li, Y., Chen, S., Liu, G., et al. (2018) Detection of pathogenic microorganisms from bloodstream infection specimens using TaqMan array card technology. Sci Rep, 8(1), 12828. https://doi: 10.1038/s41598-018-31200-3.

Figure Legend

Figure 1. Linear regression analysis. Linearity was determined by a 2-fold serial dilution of the DNA template.

Table 1. Microorganisms used in specificity assay

\begin{tabular}{lll}
\hline Organism & No. & Source/strain \\
\hline Acinetobacter baumannii & 5 & ATCC 19606; Clinical isolates (4) \\
Klebsiella pneumonia & 33 & CMCC 46117; Clinical isolates (32) \\
Escherichia coli & 8 & Clinical isolates (8) \\
Pseudomonas aeruginosa & 19 & CMCC 10104; Clinical isolates (18) \\
Enterococcus faecalis & 9 & ATCC 19433; Clinical isolates (8) \\
Enterococcus faecium & 5 & ATCC 19434; Clinical isolates (4) \\
Serratia marcescens & 7 & CMCC 41002; Clinical isolates (6) \\
Salmonella enterica & 1 & CMCC 41002 \\
Streptococcus pneumoniae & 1 & CMCC 31001 \\
Enterobacter cloacae & 15 & CMCC 43501; Clinical isolates (14) \\
Burkholderia cepacia & 13 & ATCC25416; Clinical isolates (12) \\
Enterobacter aerogenes & 1 & ATCC 13048 \\
Proteus mirabilis & 18 & BNCC 107943; Clinical isolates (17) \\
Candida albicans & 2 & CMCC 98001; Clinical isolates (1) \\
Candida glabrata & 1 & Clinical isolates (1) \\
Stenotrophomonas maltophilia & 3 & Clinical isolates (3) \\
Staphylococcus aureus & 9 & Clinical isolates (9) \\
Staphylococcus epidermidis & 3 & Clinical isolates (3) \\
Staphylococcus hominis & 1 & Clinical isolates (1) \\
Staphylococcus haemolyticus & 13 & Clinical isolates (13) \\
Staphylococcus capitis & 2 & Clinical isolates (2) \\
\hline
\end{tabular}

Table 2. Primers and probes for A. baumannii and $K$. pneumonia detection.

\begin{tabular}{lll}
\hline Name & Target Gene & Sequence $\left(5^{\prime}-3^{\prime}\right)$ \\
\hline Ab-F & OXA-51-like $\beta$-lactamase & CAC ACT ACG GGT GTT TTA \\
& $\left(\right.$ bla $a_{\text {OXA-51-like })}$ & GTT ATC CA \\
Ab-R & & CGA GCA AGA TCA TTA \\
& & CCA TAG CTT T
\end{tabular}




\begin{tabular}{lll}
\hline Name & Target Gene & Sequence (5'-3') \\
\hline Ab-Probe & & Cy5-CAA GGC CAA ACT \\
& & C-MGB \\
Kp-F & GGG CGA GGT TTA CGT \\
& Klebsiella pneumoniae & CTC AA \\
Kp-R & GCG TGT GGA TAA GAG \\
& & GTG CG \\
Kp-Probe & ROX-CCA CCA CGA GCG \\
& GC-MGB \\
\hline
\end{tabular}

Table 3. Comparison of clinical characteristics of positive patients reported by different detection methods.

\begin{tabular}{|c|c|c|c|c|}
\hline $\begin{array}{l}\text { Clinical } \\
\text { characteristics }\end{array}$ & $\begin{array}{l}\text { Positive patients by } \\
\mathrm{BC} \text { and/or ddPCR } \\
(\mathrm{n}=53)\end{array}$ & $\begin{array}{l}\text { ddPCR-only }(+) \\
(\mathrm{n}=42)\end{array}$ & $\begin{array}{l}\text { BC \& ddPCR }(+) \\
(\mathrm{n}=11)\end{array}$ & $P$ \\
\hline Age (years) & $65.7 \pm 13.3$ & $64.7 \pm 13.8$ & $69.4 \pm 11.2$ & 0.29 \\
\hline Male, n (\%) & $38(71.7)$ & $30(71.4)$ & $8(72.7)$ & 0.93 \\
\hline $\begin{array}{l}\text { Use of vasoactive } \\
\text { drugs }\end{array}$ & $35(66.0)$ & $27(64.3)$ & $8(72.7)$ & 0.60 \\
\hline $\begin{array}{l}\text { Norepinephrine, } \mathrm{n} \\
(\%)\end{array}$ & $34(64.2)$ & $26(61.9)$ & $8(72.7)$ & 0.51 \\
\hline $\begin{array}{l}\text { Epinephrine, } \mathrm{n} \\
(\%)\end{array}$ & $6(11.3)$ & $5(11.9)$ & $1(9.10)$ & 0.79 \\
\hline $\begin{array}{l}\text { Vasopressin, } \mathrm{n} \\
(\%)\end{array}$ & $12(22.6)$ & $8(19.1)$ & $4(36.5)$ & 0.22 \\
\hline $\begin{array}{l}\text { Mechnical } \\
\text { ventilation, n (\%) }\end{array}$ & $44(83.2)$ & $35(83.3)$ & $9(81.8)$ & 0.91 \\
\hline $\begin{array}{l}\text { Renal } \\
\text { replacement } \\
\text { therapy, n }(\%) \\
\text { Physical } \\
\text { examinations }\end{array}$ & $14(26.4)$ & $11(26.2)$ & $3(27.3)$ & 0.94 \\
\hline Temperature $\left({ }^{\circ} \mathrm{C}\right)$ & $38.7 \pm 0.37$ & $38.8 \pm 0.39$ & $38.6 \pm 0.21$ & 0.10 \\
\hline $\begin{array}{l}\text { Systolic blood } \\
\text { pressure (mmHg) }\end{array}$ & $86.4 \pm 17.6$ & $88.4 \pm 15.7$ & $78.6 \pm 22.8$ & 0.11 \\
\hline $\begin{array}{l}\text { Diastolic blood } \\
\text { pressure ( } \mathrm{mmHg} \text { ) } \\
\text { Complete blood } \\
\text { counts and blood } \\
\text { biochemistry }\end{array}$ & $44.2 \pm 9.57$ & $46.4 \pm 8.10$ & $41.5 \pm 7.88$ & 0.09 \\
\hline $\begin{array}{l}\text { Platelet counts, } \\
\text { median (IQR) } \\
\times 10^{9} / \mathrm{L}\end{array}$ & $61.2(54.4-93.4)$ & $83.0(63.5-108.5)$ & $40.3(15.7-103.6)$ & 0.12 \\
\hline $\begin{array}{l}\text { White blood cell, } \\
\text { median (IQR) } \\
\times 10^{9} / \mathrm{L}\end{array}$ & $10.1(7.72-13.3)$ & $10.5(8.19-13.3)$ & $8.99(3.02-26.7)$ & 0.77 \\
\hline $\begin{array}{l}\mathrm{C} \text { reactive protein } \\
(\mathrm{mg} / \mathrm{L}), \text { median } \\
(\mathrm{IQR})\end{array}$ & $152.1(125.0-185.1)$ & $140.6(110.4-179.1)$ & $204.4(150.4-277.8)$ & 0.06 \\
\hline
\end{tabular}




\begin{tabular}{|c|c|c|c|c|}
\hline $\begin{array}{l}\text { Clinical } \\
\text { characteristics }\end{array}$ & $\begin{array}{l}\text { Positive patients by } \\
\mathrm{BC} \text { and/or ddPCR } \\
(\mathrm{n}=53)\end{array}$ & $\begin{array}{l}\text { ddPCR-only }(+) \\
(\mathrm{n}=42)\end{array}$ & $\begin{array}{l}\mathrm{BC} \& \text { ddPCR }(+) \\
(\mathrm{n}=11)\end{array}$ & $P$ \\
\hline $\begin{array}{l}\text { Procalcitonin } \\
\text { (pg/L), median } \\
\text { (IQR) }\end{array}$ & $4.84(3.04-7.72)$ & $3.70(2.20-6.21)$ & $12.9(4.13-40.4)$ & 0.03 \\
\hline $\begin{array}{l}\text { Serum creatinine } \\
(\mu \mathrm{mol} / \mathrm{L}), \text { median } \\
(\mathrm{IQR})\end{array}$ & $125.6(107.2-147.1)$ & $119.2(98.9-143.6)$ & $151.5(103.9-220.9)$ & 0.22 \\
\hline $\begin{array}{l}\text { Serum lactate } \\
(\mathrm{mmol} / \mathrm{L}), \text { median } \\
(\mathrm{IQR})\end{array}$ & $3.01(2.50-3.62)$ & $2.76(2.28-3.43)$ & $4.26(2.75-6.89)$ & 0.04 \\
\hline SOFA score & $11.2 \pm 4.77$ & $10.5 \pm 4.54$ & $13.8 \pm 4.85$ & 0.03 \\
\hline APACHE II score & $23.2 \pm 7.46$ & $21.9 \pm 6.63$ & $27.9 \pm 8.77$ & 0.02 \\
\hline $\begin{array}{l}28 \text { - day } \\
\text { mortality, n (\%) }\end{array}$ & $34(64.2)$ & $24(70.6)$ & $10(90.1)$ & 0.04 \\
\hline
\end{tabular}

BC, blood culture; ddPCR, droplet digital PCR; IQR, interquartile range; SOFA, sequential organ failure assessment score; APACHE II, acute physiology and chronic health evaluation II score; Values are mean $\pm \mathrm{SD}$ or number of subjects (percentage of the column total). $P$ values for characteristics difference were calculated for comparisons by the standard normal $z$-test (means) or Fisher's exact test (proportions).

Table 4 . Analytical specificity and sensitivity of A. baumannii and K. pneumonia .

\begin{tabular}{lllllll}
\hline Microorganism & Specificity & Specificity & Sensitivity & Sensitivity & Sensitivity & Sensitivity \\
\hline & A. baumannii & K. pneumonia & Mean & SD & LoB & LoD \\
A. baumannii & $5 / 5(100)$ & $0 / 5(0)$ & 0.09 & 0.28 & 0.55 & 0.93 \\
K. pneumonia & $0 / 33(0)$ & $33 / 33(100)$ & 0.03 & 0.08 & 0.16 & 0.27 \\
Other 131 isolates & $0 / 131(0)$ & $0 / 131(0)$ & & & & \\
\hline
\end{tabular}

\begin{tabular}{llll}
\hline A. baumannii & A. baumannii & K. pneumonia & K. pneumonia \\
\hline Concentration $($ copies $/ \mu \mathrm{L})$ & CV\% & Concentration $($ copies $/ \mu \mathrm{L})$ & CV\% \\
$578.4 \pm 15.9$ & 2.7 & $551.5 \pm 12.6$ & 2.3 \\
$261.2 \pm 13.4$ & 5.1 & $240.4 \pm 8.2$ & 3.4 \\
$91.9 \pm 3.2$ & 3.5 & $90.5 \pm 5.6$ & 6.2 \\
$46.2 \pm 1.4$ & 2.9 & $62.0 \pm 2.6$ & 4.2 \\
$26.1 \pm 1.4$ & 5.3 & $36.1 \pm 3.4$ & 9.5 \\
\hline
\end{tabular}

Table 5. Analytical repeatability of A. baumannii and K. pneumonia detection $\mathrm{CV}$, coefficients of variations.

Table 6. Analytical reproducibility of A. baumannii and K. pneumonia detection

\begin{tabular}{llll}
\hline A. baumannii & A. baumannii & K. pneumonia & K. pneumonia \\
\hline Concentration (copies $/ \mu \mathrm{L})$ & CV\% & Concentration $($ copies $/ \mu \mathrm{L})$ & CV $\%$ \\
$580.1 \pm 30.6$ & 5.3 & $541.1 \pm 40.0$ & 7.4 \\
$91.0 \pm 5.5$ & 6.0 & $91.5 \pm 7.0$ & 7.6
\end{tabular}




\begin{tabular}{llll}
\hline A. baumannii & A. baumannii & K. pneumonia & K. pneumonia \\
\hline $27.1 \pm 2.0$ & 7.5 & $38.5 \pm 3.7$ & 9.7 \\
\hline
\end{tabular}

$\mathrm{CV}$, coefficients of variations.

Table 7. Clinical validation of ddPCR analysis vs . blood culture

\begin{tabular}{lll}
\hline Species & ddPCR & Blood culture \\
\hline A. baumannii & 23 & 4 \\
K. pneumonia & 26 & 7 \\
A. baumannii E K. pneumonia & 4 & 0 \\
Other microorganisms & Not detected & 22 \\
Negative & 117 & 137 \\
\hline
\end{tabular}

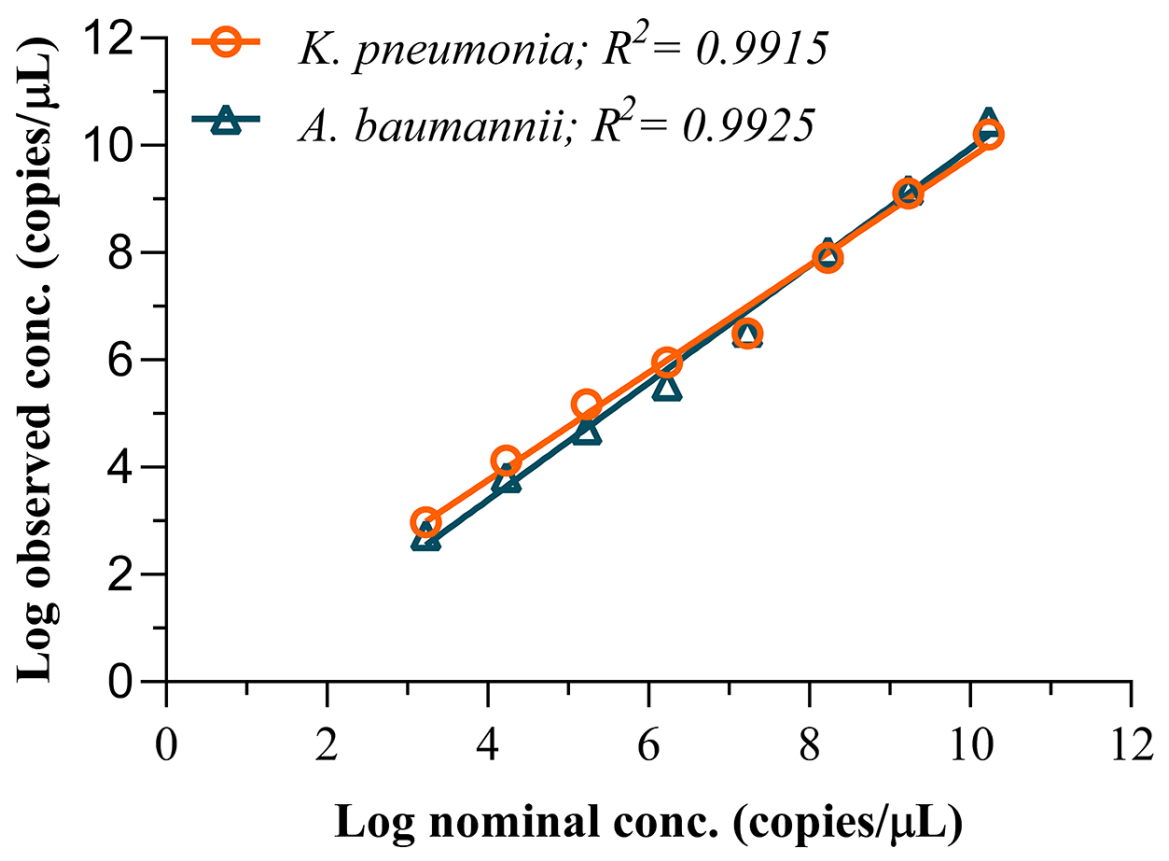

